\title{
Donepezil Treatment and Alzheimer Disease: Can the Results of Randomized Clinical Trials Be Applied to Alzheimer Disease Patients in Clinical Practice?
}

\author{
Jared R. Tinklenberg, M.D., Helena C. Kraemer, Pb.D., \\ Kristine Yaffe, M.D., Leslie Ross, Ph.D., \\ Javaid Sheikh, M.D., M.B.A., John W. Ashford, M.D., Ph.D., \\ Jerome A. Yesavage, M.D., Joy L. Taylor, Ph.D.
}

\begin{abstract}
Objective: To determine if results from randomized clinical trials of donepezil in Alzheimer disease (AD) patients can be applied to AD patients in clinical practice by comparing the findings from a Nordic one-year randomized AD donepezil trial with data from a one-year prospective, observational study of AD patients. Methods: AD patients from a consortium of California sites were systematically followed for at least one year. Their treatment regimens, including prescription of donepezil, were determined by their individual physician according to bis or her usual criteria. Results: The 148 California patients treated with donepezil had a one-year decline of 1.3 (3.5 SD) points on the Mini-Mental State Exam compared to a decline of 3.3 (4.4SD) in the 158 AD patients who received no anti-Alzheimer drugs. The Mini-Mental State Exam decline in Nordic sample was $\sim 0.25$ points for the 91 patients receiving donepezil and 2.2 for the 98 placebo patients. The overall effect sizes were estimated at about 0.49 in both studies. The California data were further analyzed using propensity methods; after taking into account differences that could bias prescribing decisions, benefits associated with taking donepezil remained. Conclusion: $A$ comparison of a randomized clinical trial of donepezil in AD patients and this observational study indicates that if appropriate methodological and statistical precautions are undertaken, then results from randomized clinical trials can be predictive with AD patients in clinical practice. This California study supports the modest effectiveness of donepezil in AD patients having clinical characteristics similar to those of the Nordic study. (Am J Geriatr Psychiatry 2007; 15:953-960)
\end{abstract}

Key Words: Propensity analyses, observational studies, donepezil effectiveness, clinical practice, Alzheimer disease

$S_{d}^{e}$ everal randomized clinical trials (RCTs) have demonstrated the efficacy and safety of donepezil and other cholinesterase inhibitors (ChEI) in treat- ing Alzheimer disease (AD) patients for periods of time up to six months. Two of these studies provided the "pivotal" phase III data for U.S. Food and Drug

Received December 7, 2006; revised March 15, 2007; accepted April 9, 2007. From the Sierra Pacific Mental Illness, Research, Education and Clinical Center, Veterans Affairs Palo Alto Health Care System, Palo Alto, CA (JRT, HCK, JS, JWA, JAY, JLT); the Department of Psychiatry and Behavioral Sciences, Stanford University, Stanford, CA (JRT, HCK, JS, JAY, JLT); the Veterans Affairs San Francisco Health Care System and the Department of Psychiatry, University of California San Francisco, San Francisco, CA (KY); and the Institute for Health and Aging, University of California San Francisco, San Francisco, CA (LR). The authors thank Sally Joseph, Pauline Luu, Art Noda, John Oehlert, and Jean Thompson for their technical assistance. Send correspondence and reprint requests to Jared R. Tinklenberg, M.D., Department of Psychiatry and Behavioral Sciences, 401 Quarry Rd., C-301, Stanford University School of Medicine, Stanford, CA 94305. e-mail: jerytink@stanford.edu

(C) 2007 American Association for Geriatric Psychiatry 
Administration (FDA) marketing approval of donepezil. ${ }^{1,2}$ However, for the practicing clinician, this research information is limited by both the short duration and by the restrictive subject selection criteria that excluded a number of patients from participating. Most clinicians are interested in practical information regarding the longer-term effects of donepezil on the patients typically seen in their practice.

A significant step toward reducing this information gap was made by a Northern European RCT of donepezil. ${ }^{3}$ This Nordic trial was longer (one year) than any previous RCTs. The issue of restrictive subject selection remains, however, because the Nordic trial retained some of the usual RCT subject exclusions. Such limitations reduce the useful information about donepezil for the many practitioners. For example, an earlier study of community-dwelling AD patients evaluated at most of the California sites collaborating in this present research study indicated that about $90 \%$ of them would be excluded from typical RCTs of anti-AD medications because of comorbid illnesses, concomitant medications, or other clinical characteristics. ${ }^{4}$ Thus, many AD patients treated by community physicians would be excluded from RCTs.

We constructed this present, prospective California study from a subset of $\mathrm{AD}$ patients who were participating in ongoing, clinical observational studies. Our aim was to determine how well information from the Nordic RCT translates to AD patients who receive their care in more general clinical settings and hence are treated with anti-AD drugs in a nonrandom fashion. An additional goal was to demonstrate the utility of signal detection-based propensity analyses in clinical observational studies. These analyses help identify any biases for certain groups of patients to receive one treatment rather than another. Identifying such biases and determining their impact on treatment outcomes permit more precise interpretations of observational studies. To facilitate comparisons with the Nordic findings, we limited this California study sample to AD patients who identified themselves as white non-Hispanics. Recent California research indicates that compared with white patients with $\mathrm{AD}$, Hispanics and other ethnic minority patients are less likely to take ChEI drugs. ${ }^{5}$

The industry-sponsored Nordic study was conducted in the late 1990s and might have been impacted by the introduction of donepezil to the mar- ket in the midst of the trial; after donepezil became available in clinical practice, there were more dropouts from the Nordic double-blind trial.

Donepezil was introduced to the California market in early 1997. Our state and Veteran's Affairs (VA)funded California study collected these data from January 1, 1998 through June 30, 2004. Thus, during the initial part of this study, practice guidelines for donepezil were not available. Many physicians were cautious about prescribing donepezil because of adverse experiences associated with tacrine (Cognex), the only other FDA-approved ChEI available at that time. Donepezil use in California increased during the study period; analyses were done to factor in these changes in prescribing patterns.

\section{METHODS}

\section{Study Design}

This study was designed to amass data from a prospective, longitudinal, multisite, observational study in California that could be directly compared to data from the Nordic RCT. ${ }^{3}$ In both studies, the diagnosis of AD was consistent with the National Institute of Neurological and Communicative Disorders and Stroke-AD and Related Disorders Association criteria for probable or possible AD and Diagnostic and Statistical Manual of Mental Disorders, Fourth Edition criteria for $\mathrm{AD}^{6,7}$ Men and women between 40 and 90 years of age were included. Patients had to have mild to moderate AD confirmed by a Mini-Mental State Exam (MMSE) score of $\geq 10$ and $\leq 26,{ }^{8}$ sufficient physical abilities to participate in the initial outpatient diagnostic process, and a caregiver who agreed to participate in the research and either lived with or closely monitored the patient. No patients could be taking donepezil or any other ChEI at their baseline assessment or during the prior four weeks.

After baseline assessment, each patient's physician determined treatment, including whether or not donepezil was prescribed according to his or her usual criteria. Patients were identified for the study after they completed a baseline assessment and a follow-up evaluation two or more months later when their treatment status was reconfirmed. All patients were expected to participate in a structured clinic reassessment about 
one year after baseline. Depending on their clinical status, some patients were seen more frequently during the study period. Patients who took any experimental drug, any other ChEI, or memantine throughout the study period were excluded from the final analyses.

\section{Study Sites}

The 11 study sites included eight Alzheimer's Disease Research Centers of California (ARCCs): Stanford/Palo Alto VA (the coordinating site), University of California Davis at Martinez, University of California Davis at Sacramento, University of California Irvine, University of California Los Angeles, University of California San Diego, CA, University of California San Francisco, and University of Southern California at Rancho, as well as three VA Mental Illness Research and Education Centers (MIRECC) in Northern California: San Francisco, Martinez, and Palo Alto.

The ARCC sites have been closely collaborating and using common research data collection protocols (Minimum Uniform Data Set [MUDS]) for over 10 years. ${ }^{9}$ Data are processed centrally through the Institute for Health and Aging at the University of California in San Francisco. To increase intersite reliability and accuracy, training and recalibration exercises are held with case reports, videos, and autopsy findings. ${ }^{10,11}$ The VA-MIRECC sites are also directed by ARCC consortium investigators and also use MUDS protocols. Patients reside in the surrounding communities and many remain under the care of their primary physicians.

All sites follow some of their patients to autopsy and systematically determine correlations between premorbid clinical diagnoses and neuropathological findings. Because of resource limitations and the realities of clinical research, however, dropouts from longitudinal study efforts are often considerable. All sites are experienced in conducting National Institutes of Health- and industry-sponsored collaborative trials of anti-AD medications. The study was part of ongoing multisite research collaborations. These are carried out in accordance with all applicable Institutional Review Board requirements.

\section{Outcome Measure}

The outcome measure of this California study was the 30-point MMSE, ${ }^{8}$ which was also used in the Nor- dic study as a secondary outcome. The MMSE has been used extensively in dementia and drug research; it provides a longitudinal "benchmark" that is understood by clinicians from different countries ${ }^{12}$ and has been evaluated psychometrically. ${ }^{13}$

\section{Statistical Analysis}

For the primary outcome measure, a $t$ test was done to test for differences between the donepezil and nodonepezil groups in one-year change. Supplementary data analyses, based on propensity methods, ${ }^{14}$ were carried out to address the observational nature of this study in which assignment to treatment is nonrandom. Propensity methods match patients on baseline characteristics that predict the propensity for an individual to receive one treatment over another. ${ }^{15}$ If, for example, patients with higher baseline MMSE scores are more likely to be prescribed donepezil than patients with lower scores, then it is important to match on baseline MMSE when contrasting treated versus untreated groups, especially in view of past studies documenting that the initial MMSE value affects rate of decline in MMSE scores. ${ }^{16}$

There are several methods for identifying which baseline characteristics predict treatment group membership, including logistic regression, discriminant analysis, and signal detection theory (SDT). We chose SDT because it can identify systematic differences in baseline characteristics, does not assume variables are normally distributed, and will identify interactions among baseline characteristics. ${ }^{17,18}$ For example, the degree to which initial MMSE predicts treatment status may depend on the patient's age. Details of the results based on propensity matching are presented below, following descriptions of the donepezil treatment and nondonepezil groups, and the overall differences in one-year outcome. Data analyses were performed using SAS version 9.1. The SDT analyses were done with receiver operating characteristic (ROC) procedures that are publicly available at http://mirecc.stanford.edu.

\section{RESULTS}

A total of 502 patients (of whom 421 [83.9\%] had probable $\mathrm{AD}$ and 81 had possible $\mathrm{AD}$ ) entered the study. Their baseline characteristics are summarized 


\begin{tabular}{|c|c|c|c|c|}
\hline & \multicolumn{2}{|c|}{ DONEPEZIL TREATMENT } & \multicolumn{2}{|c|}{ NO DONEPEZIL TREATMENT } \\
\hline & COMPLETERS & NONCOMPLETERS & COMPLETERS & NONCOMPLETERS \\
\hline $\mathrm{N}$ & 148 & 93 & 158 & 103 \\
\hline Age, years $($ mean $\pm S D)$ & $76.2 \pm 8.1$ & $76.8 \pm 7.8$ & $78.4 \pm 6.5$ & $78.6 \pm 7.5$ \\
\hline Age at symptom onset, years (mean \pm SD) & $71.6 \pm 8.8$ & $72.9 \pm 8.5$ & $73.0 \pm 7.1$ & $73.8 \pm 8.1$ \\
\hline Years of education $($ mean $\pm S D)$ & $14.1 \pm 2.7$ & $14.2 \pm 2.9$ & $13.9 \pm 3.1$ & $14.0 \pm 3.0$ \\
\hline MMSE score (mean \pm SD) & $21.0 \pm 4.2$ & $20.4 \pm 4.4$ & $19.2 \pm 4.2$ & $19.6 \pm 4.3$ \\
\hline $\mathrm{AD}$ probable, $\mathrm{n}(\%)$ & $126(85.1)$ & $74(79.6)$ & $132(83.5)$ & $89(86.4)$ \\
\hline Women, n (\%) & $91(61.5)$ & $65(69.9)$ & $96(60.8)$ & $61(59.2)$ \\
\hline VA patients, $\mathrm{n}(\%)$ & $15(10.1)$ & $7(7.5)$ & $17(10.8)$ & $9(8.7)$ \\
\hline Median date of study entry & July 11,2000 & October 31,2000 & August 3, 1999 & November 2, 2000 \\
\hline Concomitant medications, median number & 3 & 3 & 3 & 3 \\
\hline Comorbid illness, median number & 1 & 2 & 1 & 2 \\
\hline
\end{tabular}

in Table 1. At entry, concomitant medications were being taken by 212 of $241(88.0 \%)$ patients in donepezil treatment group and 217 of $261(83.1 \%)$ in nodonepezil group.

As summarized in Figure 1, 241 of the 502 patients were prescribed donepezil by their physician according to his or her usual criteria and 261 were not. At the 1-year follow-up period, 148 of the donepezil treatment group and 158 of the nondonepezil group had completed the study (Figure 1). To be a study completer, the patient needed to have an MMSE score 10 to 18 months after the baseline visit. The most common reasons for patients to not complete the study protocol were: 1 ) they did not return for an in-clinic assessment until after the 18-month end of study date; 2 ) they did have a clinic or phone assessment during the study period, but a MMSE score was not obtained because of resource or other limitations; 3 ) the donepezil treatment patients stopped taking donepezil, switched to another CHEI, or added memantinel; and 4) the no-donepezil treatment patients started taking a CHEI or started an experimental drug.

In investigating completer versus noncompleter biases using ROC analyses, we found that the biases were primarily due to time of study entry and site factors rather than clinical characteristics. Specifically, one site had a disproportionate number of noncompleters in both donepezil and no-donepezil groups; at this site, no-donepezil subjects were more likely to be a study completer if their baseline visit occurred prior to 2001. Analyses indicated that inclusion of this particular site did not bias overall results. In view of the fact that there were no significant clinical differences between completers and noncom-

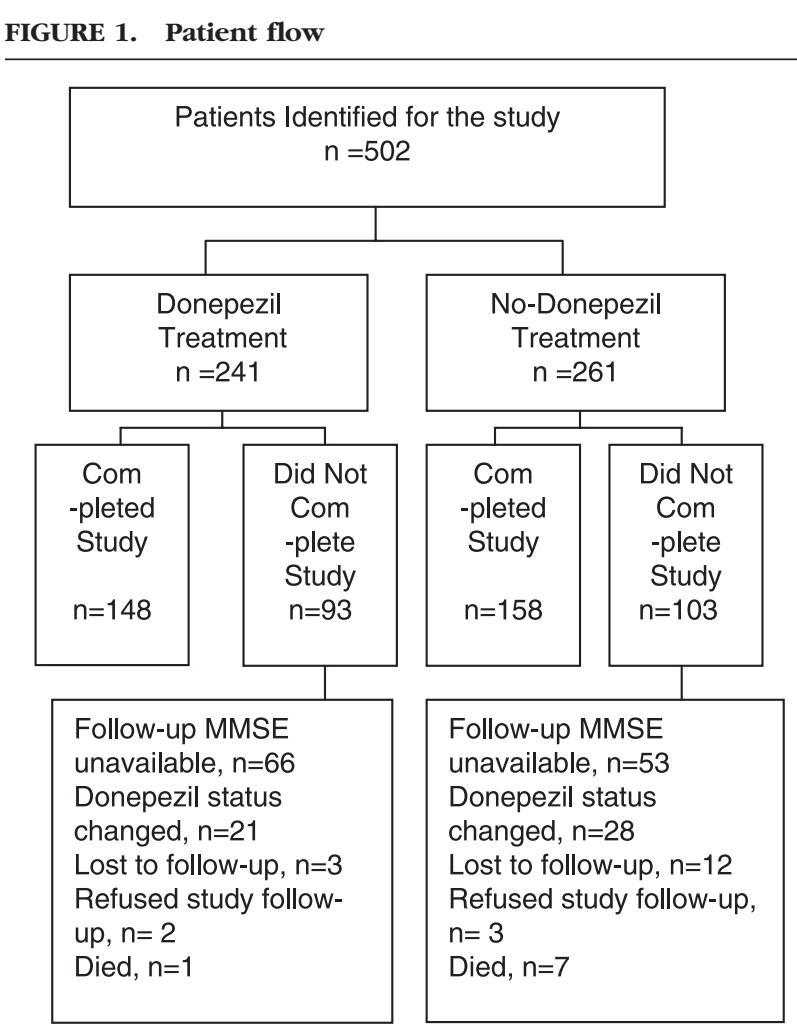

pleters, the remainder of the results will focus on the 306 study completers.

\section{Comparison of Donepezil and No-Donepezil Groups}

As indicated in Figure 2, the 148 California patients who completed the study and received donepezil treatment had an average one-year decline of 
FIGURE 2. California Observational AD Study Versus the Nordic Randomized Clinical Trial

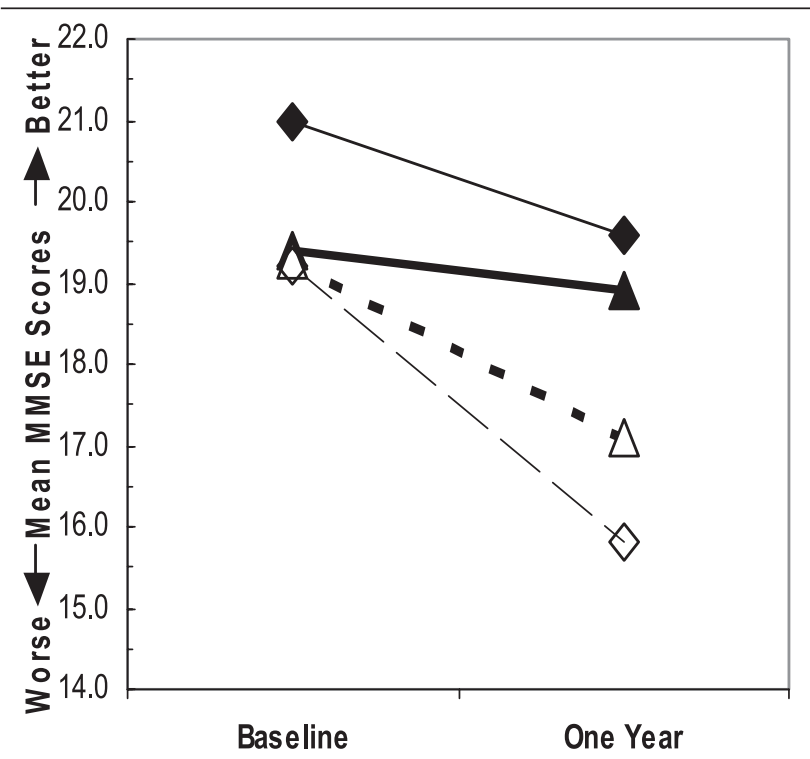

Notes: Shown are Mini-Mental State Exam scores at baseline and 1 year for four groups. California patients prescribed donepezil who were study completers $(\mathrm{N}=148$, thin solid line), Nordic patients treated with donepezil who were study completers $(\mathrm{N}=91$, thick solid line), Nordic patients treated with placebo who were study completers $(\mathrm{N}=98$, thick broken line), and California patients not prescribed donepezil who were study completers $(\mathrm{N}=158$, thin broken line).

1.3 points ( $3.5 \mathrm{SD}$ ) on the MMSE compared to a 3.3-point (4.4 SD) decline in the 158 completers who received no donepezil or other anti-AD drugs during the study period. This difference was statistically significant $\left(t^{304}=-4.32, \mathrm{p}<0.0001\right)$. By comparison, the annualized MMSE decline in Nordic sample was about 0.25 for the 91 donepezil patients who completed the trial and about 2.2 for 98 placebo completers. The overall effect sizes of these treatmentassociated differences were estimated at about 0.49 in both the California and the Nordic studies. ${ }^{19}$

\section{Propensity Analyses}

Despite the comparable effect sizes of the two studies, it is important to identify biases in nonrandomized studies that might affect whether patients were in a given treatment group or not. In this present study, for example, patients who were prescribed donepezil had baseline MMSE scores that were 1.8 points higher on average than those who were not prescribed donepezil.
In addition, patients were somewhat less likely to be prescribed donepezil regardless of their clinical characteristics in the earlier years of this study when donepezil prescribing was less widespread. As described below, a modest donepezil-associated benefit remained when these and other baseline differences were taken into account.

The first step of the propensity analysis was to identify baseline characteristics that significantly influenced the likelihood or propensity of whether or not donepezil was prescribed. We used ROC classification tree methods with a $\mathrm{p}$ value of $<0.01$ to identify variables suggested by the literature that might explain whether or not they were prescribed donepezil. These 27 variables included both patient characteristics, such baseline cognitive status (MMSE), age of disease onset, comorbid illnesses, concomitant medications, years of education, sex, marital status, relationship with caregiver, living arrangement, and veteran status, as well as nonpatient characteristics, such as date of baseline assessment and study site.

The ROC tree analysis indicated that the first (strongest) branching variable predicting donepezil prescription was the date of the baseline assessment; patients prescribed donepezil were more likely to have their baseline in 2000 or later. There was a second branch within the group seen before 2000, in which patients who had no significant comorbid illnesses were more likely to be prescribed donepezil than those who did. The ROC identified no other significant variables predicting donepezil prescription.

We then stratified all patients into the above three propensity groups that differed in characteristics associated with donepezil prescribing; 1) patients seen in 2000 or later; 2) patients seen before 2000 who had no comorbid illnesses; and 3) patients seen before 2000 with one or more comorbid illnesses. A total of $59.3 \%$ (96 of 162) patients in propensity group 1 were prescribed donepezil, 53\% (28 of 53) in group 2, and only $26 \%$ (24 of 91$)$ in group 3.

In each propensity group, patients who received donepezil declined less than patients who were not prescribed donepezil. The estimated treatment effect size was 0.37 for propensity group $1,0.19$ for propensity group 2 , and 0.79 for propensity group 3 . A $2 \times 3$ analysis of variance was used to compare differences in MMSE decline across the six groups (donepezil versus no donepezil, stratified by propensity). The differences in rate of decline according to the propensity grouping 
were not significant $\left(F^{2,300}=2.10, p=0.12\right)$. The difference in decline associated with donepezil was significant $\left(\mathrm{F}^{1,300}=12.39, \mathrm{p}<0.001\right)$. Third, the propensity $\times$ donepezil interaction was not significant $(\mathrm{F}=1.66, \mathrm{p}=$ 0.19 ), indicating that the benefit associated with donepezil was comparable within groups of $\mathrm{AD}$ patients matched for their likelihood of being prescribed donepezil. Taken together, these results indicate that the modest benefit associated with taking donepezil remained after taking into account the lack of randomization to treatment.

\section{DISCUSSION}

This one-year observational study supports the effectiveness of donepezil treatment on cognitive function in some California $\mathrm{AD}$ patients who were selected to have clinical and ethnic characteristics similar to patients in the Nordic randomized clinical trial. ${ }^{3}$ The magnitude of the positive effects with donepezil was smaller in the California study: the one-year MMSE decline in California sample was $\sim 1.3$ for patients prescribed donepezil and $\sim 0.25$ for Nordic patients receiving donepezil. However, the effect size of the differences between donepezil versus no donepezil in both studies was about 0.49 , or one-half of a standard deviation. Thus, our analyses indicated that results from the Nordic RCT can be predictive with AD patients in clinical practice if appropriate methodological and statistical precautions are considered. The differences in rate of MMSE decline between the two studies may result from a combination of factors such as differences in concomitant medications, comorbid conditions, frequency of monitoring, and medication compliance.

In this California study, the patients and their caregivers knew that donepezil and other drugs would be prescribed in accordance with their physician's usual clinical practices. This meant that some California patients received medications with anticholinergic or other psychoactive properties that could interfere with the effects of donepezil. ${ }^{20}$ The Nordic patients knew their study medications would be randomly assigned, and their use of other medications would be restricted. The California patients also were less restricted in terms of comorbid medical problems; they could have other illnesses if their physician felt they might benefit from donepezil treatment. The Nordic trial excluded certain medical conditions such as obstructive pulmonary disease and asthma.

Some California patients had one or more interim clinic visits or phone follow-ups before their one-year reassessment, but in general, they received less monitoring and less frequent repeated administrations of the MMSE, with its known practice effects, than the Nordic patients. ${ }^{21}$ In the Nordic study, the patients agreed to four scheduled in-clinic follow-up assessments and they knew they would receive randomly either donepezil or placebo. ${ }^{3}$ With less scheduled attention, the California patients who were prescribed donepezil may have been less compliant. Within the California study, patients prescribed donepezil were more likely to have interim clinic visits than those who did not receive donepezil. This could have contributed to their somewhat better 1-year scores. These various considerations may explain the differences found between the Nordic and California results.

These differences can also be considered in the context of a randomized, placebo-controlled one-year U.S. clinical trial of donepezil that had more restrictive entry criteria than either the California or Nordic studies. ${ }^{22}$ The MMSE scores for both donepezil and placebo groups were generally better throughout this U.S. trial than in the Nordic or California studies. These MMSE variations underscore the importance of methodological details in interpreting study results.

One limitation of this California study was the possibility of biases due to the high dropout rate. In all, $39 \%$ of the California patients did not complete the study. The Nordic study had a noncompletion rate of about $33 \%$, with the largest portion of dropouts occurring later in the trial when donepezil became clinically available. In this California study, study completers and noncompleters did not differ significantly in their baseline clinical characteristics. Although there were two nonclinical factors associated with completing the study-site differences and time of study entry-analyses indicated that these factors did not bias overall results. Taken together, these findings indicate it is unlikely that the factor of noncompleters significantly impacted the California results. We did not perform "intention-to-treat" analyses in this study because, in conditions like Alzheimer disease where deterioration over time is likely, carrying forward the last observation is problematic. Interpreting differences between active and placebo 
groups is especially problematic if treated patients tend to drop out early because of unpleasant side effects and relatively high cognitive scores are carried forward to the endpoint. The side effects reported by patients prescribed donepezil were similar to those reported in the Nordic and other studies: mainly nausea or other gastrointestinal symptoms, mild depression, and vague anxiety or agitation.

An additional limitation of this California study is nonrandomness of prescribing, in that each patient's physician decided by his or her usual criteria whether or not to treat with donepezil. Nonrandom prescribing may result in two groups that differ substantially in either clinical or nonclinical factors, which may in turn lead to a biased estimate of a treatment effect. To gain a clearer picture, not biased by baseline differences, we conducted propensity analyses. ${ }^{14,15}$ Propensity analyses have similarly been used in cardiology and cancer research to examine patient outcomes in clinical practice, when matching on key baseline characteristics is also important. $^{23}$ When we performed these propensity analyses, the statistical significance of the donepezilassociated benefit remained. The effect size of donepezil, which was estimated at about 0.49 in both the California and the Nordic studies, may be viewed as small to moderate in terms relevant to clinical practice. ${ }^{24}$ A related limitation of this California study is that MMSE testing was not done blind to treatment status, as is done in randomized clinical trials. The effect size is often exaggerated in observational studies, including nonblinded studies, compared to randomized clinical trials that address the same research question. ${ }^{25}$ Importantly, the effect sizes in this California observational study were not larger those of the Nordic randomized clinical trial.

One advantage of this observational AD treatment study is that the results probably generalize into a typical clinical practice more directly than results from RCTs. RCTs often have exclusionary criteria that screen out a number of patients typically seen in ordinary clinical practice. ${ }^{4,26}$ In addition, some patients who fulfill all entry criteria for a RCT are unwilling to participate in a RCT where they may not receive active medications. ${ }^{27}$ It is usually easier to enroll a more representative sample of patients into studies where they are assured of getting whatever treatment their individual physician thinks is best for them. Thus, more representative patient samples in observational studies may partially offset the inherent advantages of RCTs in which most biases are intrinsically minimized.

Consequently, observational studies can provide useful information on what a typical physician can expect in his or her clinical practice if appropriate procedures, such as propensity analyses, are utilized to assure that nonrandom factors do not significantly bias the findings. When we completed these analyses, our one-year California study indicated that donepezil treatment does have modest effectiveness in AD patients having clinical and ethnic characteristics similar to those of the Nordic RCT.

\section{LIST OF CONTRIBUTORS}

Alzheimer's Disease Research Centers of California: University of California Davis-Martinez: B. Reed, C. Bibeau, J. Coleman, and J. Webb; University of California Davis-Sacramento: D. Mungas, C. DeCarli, W. Jagust, B. Henk, and M. Verma; University of California Irvine: C. Cotman, R. Mulnard, M. Dick, C. Kawas, and H. Kim; University of California Los Angeles: J. Cummings, J. Ringman, M. Carter, K. Metz, and L. Brendt; University of California San Diego: D. Galasko, D. Salmon, C. Robinson, and M. Sundsmo; University of California San Francisco: B. Miller, K. Yaffe, J. Johnson, J. Kramer, and R. Gearheart; University of Southern California-Rancho Los Amigos: H. Chui, F. Segal-Gidan, B. Smith, and A. Ireland; Stanford/Palo Alto VA (coordinating site): H. Davies, J. Kim, H. Leutwyler, P. Luu, C. McFeeters, L. Newkirk, J. Oehlert, and J. Thompson; Institute of Health and Aging \& Alzheimer's Disease Program of California: P. Fox, D. Tyrrell, C. Motylewski-Link, R. Chapman, M. Lackey, and P. Tang; Veteran Affairs Mental Illness Research and Education Centers in Northern California: Martinez: B. Reed, C. Bibeau, and J. Webb; Palo Alto: J. Yesavage, E. Gere, and R. O'Hara; San Francisco: K. Yaffe, E. Edwards, C. O'Shea, and P. Sayegh

This work was supported by the State of California, Department of Health Services (grant 03-75273), VA 
Sierra Pacific Mental Illness Research and Education

Centers, and by the National Institute on Aging

(AG17824).
Presented at the 10th International Conference on Alzheimer's Disease and Related Disorders, Madrid, Spain, July 16-21, 2006.

\section{References}

1. Rogers SL, Doody RS, Mohs RC, et al: Donepezil improves cognition and global function in Alzheimer's disease: a 15-week, double-blind, placebo-controlled study. Arch Intern Med 1998; 158 : 1021-1031

2. Rogers SL, Farlow MR, Doody RS, et al: A 24-week, double blind, placebo-controlled trial of donepezil in patients with Alzheimer's disease. Neurology 1998; 50:136-145

3. Winblad B, Engedal K, Soininen $\mathrm{H}$, et al, and the Donepezil Nordic Study Group: a 1-year, randomized, placebo-controlled study of donepezil in patients with mild to moderate AD. Neurology 2001; 57:489-495

4. Schneider LS, Olin JT, Lyness SA, et al: Eligibility of Alzheimer's disease clinic patients for clinical trials. J Am Geriatr Soc 1997; 45:923-928

5. Mehta KM, Yin M, Resendez C, et al: Ethnic differences in acetylcholinesterase inhibitor use for Alzheimer disease. Neurology 2005; 65:159-162

6. McKhann G, Drachman D, Folstein M, et al: Clinical diagnosis of Alzheimer's disease: report of the NINCDS-ADRDA Work Group under the auspices of the Department of Health and Human Services Task Force on Alzheimer's Disease. Neurology 1984; 34:939-944

7. American Psychiatric Association: Diagnostic and Statistical Manual of Mental Disorders, 4th Ed. Washington, DC, American Psychiatric Association, 1994

8. Folstein MF, Folstein SE, McHugh PR: "Mini-Mental State": a practical method for grading the cognitive state of subjects for the clinician. J Psychiatr Res 1975; 12:189-198

9. Edwards ER, Lindquist K, Yaffe K: Clinical profile and course of cognitively normal patients evaluated in memory disorders clinics. Neurology 2004; 62:1639-1642

10. Chui HC, Victoroff JI, Margolin D, et al: Criteria for the diagnosis of ischemic vascular dementia proposed by the State of California Alzheimer Disease Diagnostic and Treatment Centers. Neurology 1992; 42:473-480

11. Chui HC, Mack W, Jackson JE, et al: Clinical Criteria for the Diagnosis of Vascular Dementia: A multicenter study of comparability and interrater reliability. Arch Neurology 2000; 57:191196

12. Salmon DP, Thal LJ, Butters N, et al: Longitudinal evaluation of dementia of the Alzheimer's type: a comparison of three standardized mental status examinations. Neurology 1990; 40:12251230

13. Mungas D, Reed BR: Application of item response theory for development of a global functioning measure of dementia with linear measurement properties. Stat Med 2000; 19:1631-1644

14. Rubin DB: Estimating causal effects from large data sets using propensity scores. Ann Intern Med 1997; 127:757-763

15. D'Agostino RB Jr: Propensity score methods for bias reduction in the comparison of a treatment to a non-randomized control group. Stat Med 1998; 17:2265-2281

16. Mendiondo MS, Ashford JW, Kryscio RJ, et al: Modeling Mini Mental State Examination changes in Alzheimer's disease. Stat Med 2000; 19:1607-1616

17. Kiernan M, Kraemer HC, Winkleby MA, et al: Do logistic regression and signal detection identify different subgroups at risk? Implications for the design of tailored interventions. Psychol Meth 2001; 6:35-48

18. Kraemer HC, Lowe KK, Kupfer DJ: To Your Health: How To Understand What Research Tells Us About Risk. New York, Oxford University Press, 2005

19. Cohen J. Statistical Power Analysis for the Behavioral Sciences. New York, Academic Press, 1969

20. Huey ED, Taylor JL, Luu PA, et al: Factors associated with use of medications with potential to impair cognition or cholinesterase inhibitors among Alzheimer's disease patients. Alzheimer Dement 2006; 2:314-321

21. Galasko D, Abramson I, Corey-Bloom J, et al: Repeated exposure to the Mini-Mental State Examination and the Information-MemoryConcentration Test results in a practice effect in Alzheimer's disease. Neurology 1993; 43:1559-1563

22. Mohs RC, Doody RS, Morris JC, et al: A 1-year placebo controlled preservation of function survival study of donepezil in $\mathrm{AD}$ patients. Neurology 2001; 57:481-488

23. Winkleby MA, Flora JA, Kraemer HC: A community-based heart disease intervention: predictors of change. Am J Public Health 1994; 84:767-772

24. Kraemer HC: Reporting the size of effects in research studies to facilitate assessment of practical or clinical significance. Psychoneuroendocrinology 1992; 17:527-536

25. Ioannidis JP. Contradicted and initially stronger effects in highly cited clinical research. JAMA 2005;294:218-228

26. Humphreys K, Weisner C: Use of exclusion criteria in selecting research subjects and its effect on the generalizibility of alcohol treatment outcome studies. Am J Psychiatry 2000; 157:588-594

27. Rush AJ, Trivedi MH, Wisniewski SR, et al: Bupropion-SR, sertraline or venlafaxine-XR after failure of SSRIs for depression. N Engl J Med 2006; 354:1231-1242 